\title{
Biofunctionalized 3D Nanopillar Arrays Fostering Cell Guidance and Promoting Synapse Stability and Neuronal Activity in Networks
}

\author{
Hayder Amin, ${ }^{* \dagger \odot}$ Michele Dipalo, ${ }^{\ddagger \odot}$ Francesco De Angelis, ${ }^{\ddagger \odot}$ and Luca Berdondini ${ }^{\dagger}$
}

${ }^{\dagger}$ Nets ${ }^{3}$ Laboratory, Department of Neuroscience and Brain Technologies (NBT), and Department of Plasmon Nanotechnologies, Fondazione Istituto Italiano di Tecnologia (IIT), Via Morego 30, 16163 Genoa, Italy

\section{Supporting Information}

\begin{abstract}
A controlled geometry of in vitro neuronal networks allows investigation of the cellular mechanisms that underlie neuron-to-neuron and neuron-extracellular matrix interactions, which are essential to biomedical research. Herein, we report a selective guidance of primary hippocampal neurons by using arrays of three-dimensional vertical nanopillars (NPs) functionalized with a specific adhesion-promoting moleculepoly-DL-ornithine (PDLO). We show that $90 \%$ of neuronal cells are guided exclusively on the combinatorial PDLO/NP substrate. Moreover, we demonstrate the influence of the interplay between nanostructures and neurons on synapse formation and maturation, resulting in increased expression of postsynaptic density-95 protein and enhanced network cellular

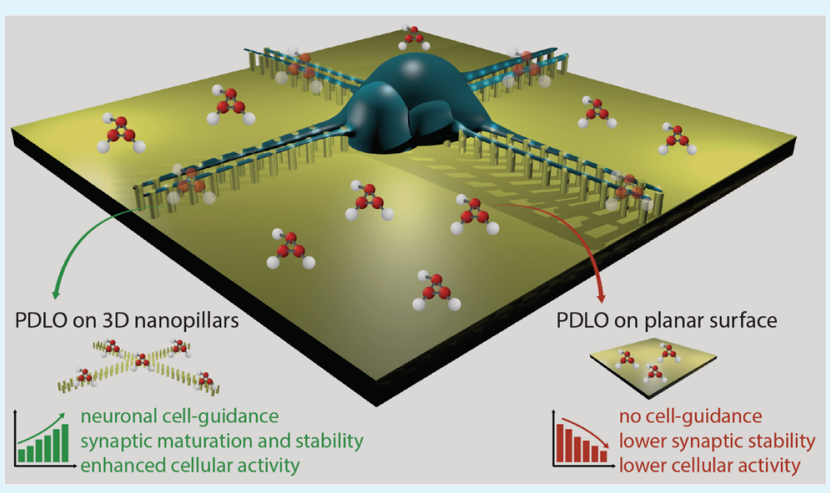
activity conferred by the endogenous c-fos expression. Successful guidance to foster synapse stability and cellular activity on multilevel cues of surface topography and chemical functionalization suggests the potential to devise technologies to control neuronal growth on nanostructures for tissue engineering, neuroprostheses, and drug development.
\end{abstract}

KEYWORDS: 3D nanopillar arrays, surface functionalization, cell guidance, PDLO, primary hippocampal neurons, synapse stability

\section{INTRODUCTION}

The function of the nervous system relies on the complex architecture of the extraordinary number of neuronal subtypes. ${ }^{1}$ The complexity of human behaviors is determined by the coordination of globally integrated activity of neuronal ensembles, which is itself mediated by the activity-dependent changes in synaptic efficacy. ${ }^{2}$ Neuronal cells use electricity to control their physical and chemical functions and modulate synaptic activity. ${ }^{3}$

Dysregulation of electrical and synaptic activities has been found in different neurological disorders. ${ }^{4-6}$ In vivo, cellular responses such as proliferation, differentiation, migration, and apoptosis $^{7}$ are influenced by the competitive external biochemical and physical guidance cues in the neuronal microenvironment, ${ }^{8}$ of which the extracellular matrix (ECM) is one of the main components. ${ }^{9}$ However, the investigation of a defined set of physical and chemical neuronal guidance cues that foster synapse maturation and stability is essential but has remained so far incomplete.

Thus, it is of great interest in neuroscience, tissue engineering, and regenerative medicine to adopt a reductionist in vitro model implemented as artificial niches to understand multiple cellular mechanisms of cell-to-cell and cell signaling pathways. In turn, this model is essential to provide a better understanding of basic research studies and pathologies as well as investigating cellular responses for building neuroprosthetic scaffolds and drug development.

Recently, progress in cell culture and microfabrication techniques has enabled in vitro cell-based sensor devices to circumvent the complex in vivo hierarchical micro/nanotopographies of the neuronal environment. ${ }^{10}$ Therefore, surface chemistry combined with micro/nanofabrication techniques $^{11,12}$ has been developed to precisely pattern the cellular growth with high spatial control to study cell responses and functions under defined conditions. ${ }^{13-15}$ Also, many fabrication methods have been reported ${ }^{16}$ to employ a range of different surface topographical nanostructures such as nanofiber, nanorods, nanotubes, nanopillars (NPs), and nanowires, ${ }^{17}$ developed for molecular delivery, ${ }^{18}$ probing enzymatic activity, ${ }^{19}$ and cell diagnosis of cytoskeleton state. ${ }^{20}$ These nanostructures have been fabricated with different materials and interfaced with various cell line models, ${ }^{21,22}$ immune cells, ${ }^{23}$ and primary neurons. $^{24}$ Furthermore, it is vital to investigate the morphological and electrophysiological changes of neuronal ensembles in well-defined cell configurations. Thus, cell patterns were also implemented on multielectrode arrays allowing simultaneous recording of electrophysiology-based

Received: January 8, 2018

Accepted: April 5, 2018

Published: April 5, 2018 
extracellular activity and tracking the signal propagation between cells in well-defined neuronal networks. ${ }^{25,26}$ Importantly, guiding cells and their processes along defined geometric nanotopographical patterns allows insight into the specific mechanisms underlying the physical cues of cellular networks and provides a key step for understanding neuronal circuit formation. In turn, this would enable the engineering of scaffolds for brain-controlled prosthetic devices. ${ }^{29}$ Previously, considerable efforts have been put forth to demonstrate the capability of three-dimensional (3D) vertical nanostructures to enhance cell adhesion and survival, ${ }^{28}$ accelerate polarization, ${ }^{29}$ and record extracellular and intracellular cellular activity. ${ }^{30-34}$ Although, understanding the synergistic effect of topographical and chemical cues on neurite guidance has been substantially investigated, ${ }^{35}$ assessing the combination of physical cues (3D NPs) and specific chemical cues (surface functionalization) to stimulate the synaptic network formation, maturation, and stability has lagged behind. Furthermore, although previous studies reported on the geometrical restriction of neuronal ensembles by physical and chemical modifications, our work sought to engineer a unique simplified patterning method to foster, rather than force guidance of any part of the neuronal ensemble on nanostructured substrates.

Here, we report a simple yet robust method for guiding the growth of hippocampal neuronal cells in a defined architecture without imposing constraints and enabling neuronal network features to function naturally. We demonstrate the possibility to use 3D vertical NPs functionalized with a specific polycationic adhesion-promoting molecule poly-DL-ornithine (PDLO) to generate an autonomic self-patterned array of neuronal ensembles. We find that this localized combinatorial substrate, without prerequisite cell-loading procedure and without material-driven restraints, fosters geometrically optimized cell attachment and patterning. We demonstrate that this combinatorial substrate enhances the synaptic formation and maturation and promotes synapse stability and network cellular activity. The self-ordered array of adhered neurons may have the potential to decipher the biological mechanisms of their molecular and cellular properties and to explore drug delivery by which neurons recognize nanotopographies.

\section{EXPERIMENTAL SECTION}

2.1. Fabrication of 3D NP Arrays. 3D gold $(\mathrm{Au})$ NPs were fabricated on a silicon nitride supporting membrane using ion beam milling as reported in our previous work..$^{31,36}$ Briefly, a layer of resist polymer was deposited on the silicon nitrate membrane by spincoating. The cylindrical structure of the NPs was defined by focused ion beam milling from the backside of the membrane using a gallium ion source. A high dose of secondary electrons was the resultant of the gallium ions and the resist interaction, which in turn led to the inversion of an exposed thin layer of the polymer surrounding the milled region. Subsequently, when the sample was immersed in a solvent, the unexposed resist was removed, whereas the exposed one remained on the sample, hence forming NPs. The size of the devised cylindrical NPs was $1.8 \mu \mathrm{m}$ (height) and $150 \mathrm{~nm}$ (diameter width) coated with a thin Au layer $(25 \mathrm{~nm})$, and the location of those NPs could be precisely controlled. Typically, we patterned those 3D NPs in $9 \times 9$ square arrays with $2 \mu \mathrm{m}$ spacing in long stripes, and we also tested the growth of neurons on a ring-shaped circle with $10 \mu \mathrm{m}$ diameter.

2.2. 3D NP Array Coating Preparation. Each NP substrate (10 $\times 10 \mathrm{~mm}^{2}$ ) was fixed on an $18 \mathrm{~mm}$ glass coverslip that was placed in a $35 \times 10 \mathrm{~mm}$ Petri dish (Corning). The samples were cleaned with oxygen plasma and sterilized by ethanol $70 \%$ for $15 \mathrm{~min}$. Next, the samples were washed $4 \times$ in sterile double-distilled water (DDW) and left to dry under a sterile laminar flow hood. The samples were preconditioned by overnight incubation at $37{ }^{\circ} \mathrm{C}$ and $5 \% \mathrm{CO}_{2}$ with complete neurobasal medium (CNM) containing $2 \%$ B-27, $1 \%$ penicillin/streptomycin, and 1\% GlutaMax Supplements (all reagents from Life Technologies). On the next day, CNM was removed, and the samples were immediately coated with $70 \mu \mathrm{g} / \mathrm{mL}$ PDLO (SigmaAldrich) and incubated for $8 \mathrm{~h}$ at $37{ }^{\circ} \mathrm{C}$ and $5 \% \mathrm{CO}_{2}$. The samples were then washed $4 \times$ in sterile DDW and left to dry under the hood before cell seeding.

2.3. Hippocampal Neuronal Culture. Embryonic hippocampal neurons were isolated at day 18 (E18) from Sprague-Dawley rats (Charles River Laboratories) according to our previously published study ${ }^{4}$ Briefly, embryos were removed and decapitated, and the brains were extracted from the skulls. We placed the brains in cold Hanks balanced salt solution, and after dissection, hippocampi were placed for $30 \mathrm{~min}$ in $0.125 \%$ trypsin-ethylenediaminetetraacetic acid and incubated at $37{ }^{\circ} \mathrm{C}$ in a water bath to dissociate the tissue. We then blocked the trypsin activity by CNM, supplemented with $10 \%$ fetal bovine serum (FBS), and centrifuged the tubes for $5 \mathrm{~min}$ at $1200 \mathrm{rpm}$. The supernatant was discarded, and fresh CNM and 10\% FBS were added. The hippocampi were dissociated gently by pipetting and the solution was filtered through a cell strainer and centrifuged for $7 \mathrm{~min}$ at $700 \mathrm{rpm}$. The supernatant was discarded, and the cells were resuspended in CNM and counted using trypan blue and a hemocytometer. We seeded the cells in $50 \mu \mathrm{L}$ drops to reach the final low density of $500 \mathrm{cell} / \mathrm{mm}^{2}$ on the coated NP substrates and then incubated at $37{ }^{\circ} \mathrm{C}$ with $5 \% \mathrm{CO}_{2}$ and $95 \%$ humidity. After $1 \mathrm{~h}$, we added $2 \mathrm{~mL}$ of CNM to the culture chamber and incubated under the same conditions. For maintenance and cell culture growth, one-third of the medium was routinely replaced with fresh CNM every 4 days. All reagents were obtained, unless indicated differently, from Life Technologies. Dissociated neurons were seeded on sample substrates at low density, that is, $500 \mathrm{cell} / \mathrm{mm}^{2}$, without any glia-supportive cells, and exhibited persistent survival on the network growth pattern for long term, until cultures were used for experiments at 21 days in vitro when mature functional synapses were formed. Neurons were maintained in neurobasal media supplemented with B-27, penicillin/ streptomycin, and GlutaMax (Life Technologies), and one-third of the media was routinely exchanged every 4 days.

2.4. Immunofluorescence Protocol. We performed immunostaining on rat hippocampal cultures grown on multiple substrates [PDLO/NPs, poly-L-ornithine (PLO)/NPs, poly-L-lysine (PLL)/NPs, poly-D-lysine (PDL)/NPs, PDLO/flat Au, PDLO/glass, and PDL/ glass] using a previously described protocol. ${ }^{37}$ Briefly, we removed the culture medium and washed the sample in phosphate-buffered saline (PBS-1X) (Life Technologies) at $37{ }^{\circ} \mathrm{C}$, and then, we fixed the samples in paraformaldehyde (4\% in PBS-1X) for $15 \mathrm{~min}$ at room temperature $(\mathrm{RT})$. We washed the sample $4 \times$ with PBS- $1 \times$; then, the cells were permeabilized with $0.1 \%$ Triton X-100 in PBS- $1 \times$ (PBST) for $10 \mathrm{~min}$. The cells were blocked with 5\% normal goat serum (NGS) (EuroClone) for $1 \mathrm{~h}$ before incubation with primary antibodies. The cells were then incubated overnight at $4{ }^{\circ} \mathrm{C}$ in primary antibodies diluted in the NGS blocking buffer. The following primary antibodies were used: guinea pig anti-MAP2 (Synaptic Systems; 1:1000), rabbit anti-c-fos (Calbiochem Millipore; 1:500), and mouse anti-postsynaptic density-95 (PSD-95) (cell signaling; 1:100). We performed three consecutive washing steps in PBST for 5 min per wash, and the cells were incubated for $1 \mathrm{~h}$ in the dark at RT with the corresponding secondary antibodies, including Alexa Fluor 488, Alexa Fluor 546, and Alexa Fluor 647 (all from Life Technologies; 1:1000). Subsequently, the cells were washed $4 \times$ with PBST and incubated for $15 \mathrm{~min}$ in the dark at RT with the nuclear marker Hoechst 33342 (Thermo Fisher Scientific; 1:500) diluted in PBS- $1 \times$. We visualized and acquired all images with $20 \times$ and $40 \times$ objective lenses using a Leica SP5 upright confocal microscope (Leica Microsystems).

2.5. Image Analysis. Granulometric filtering method was performed to quantify the PSD-95 assembles in various functionalization conditions as previously reported. ${ }^{37}$ Briefly, we converted the images to grayscale to enhance the contrast between signal and noise, and then, we set a manual threshold. Next, in a composite background 
image, we identified the small grains of the PSD-95 construct based on size and shape, which consists of several pixels. Then, by using ImageJ analysis software platform, ${ }^{38}$ we performed integral intensity thresholding to assign the granulometric filtering intensities. We set an arbitrary offset, that is, a dashed line at 30 to pinpoint the PSD-95 puncta above that level, whereas the yellow bars represent the location of the puncta above the offset. Furthermore, we quantified the density of PSD-95 construct by using an automatic particle counting method integrated into ImageJ platform. ${ }^{38}$ Thus, we analyzed the synaptic density by counting immunoreactive PSD-95 puncta per $\mu \mathrm{m}^{2}$ visualized in multiple fields of the images in multiple cultures. In addition, we quantified the intensity of c-fos immunofluorescence by using an automatic object counting method integrated into ImageJ platform, which allows employing a polygon segmentation to generate polygon-shaped regions of interests (ROIs) from single cells indicating the expression of c-fos intensity. ${ }^{4,38}$

\section{RESULTS}

3.1. Cell Guidance. To investigate the effect of the combinatorial functionalized PDLO-3D NPs (PDLO/NPs) on promoting defined geometric patterning of cell growth, neuronal cultures were fixed with $2 \%$ glutaraldehyde solution in deionized water for $40 \mathrm{~min}$ at RT and processed for scanning electron microscopy (SEM) acquisition (FEI NanoLab 600 dual beam system) as previously described. ${ }^{39}$ SEM images evinced both the cell body and neurites of hippocampal neurons guided on the PDLO/NPs (Figure 1). The neurites

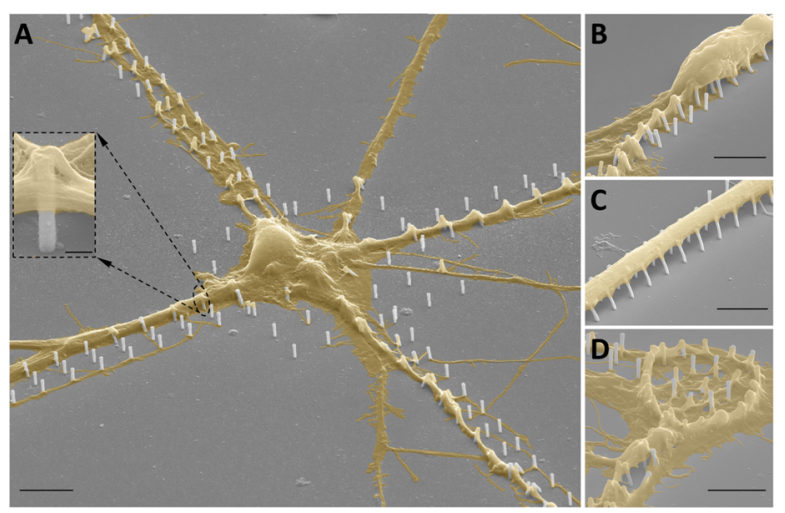

Figure 1. Colored SEM images of the autonomous neuronal cell guidance on biofunctionalized 3D NPs. (A) Cell body and extended neurites of hippocampal neuronal culture are guided by the geometrical pattern of PDLO/NPs. The inset displays a closer view of the tight adhesion of the neuritic membrane wrapping around the biofunctionalized 3D NPs. Scale bar represents $500 \mathrm{~nm}$. (B) Close-up image of a cell body and neuritic extensions showing anchoring on PDLO/NPs. (C) Bundle of neuronal axons is precisely guided on PDLO/NPs. (D) Neuronal axons are guided along a complex circularlike pattern of NPs functionalized with PDLO. Scale bars represent 4 $\mu \mathrm{m}$ for $(\mathrm{A}-\mathrm{D})$.

were aligned on PDLO/NPs in regions away from the soma, which indicates that PDLO/NPs foster the polarization of those neurites. Despite having coated the whole substrate with PDLO, that is, on NPs and outside, neurons and their projections were selectively attached and grown only on the $\mathrm{PDLO} / \mathrm{NPs}$ compared to the exterior regions of the PDLO/ planar surface (Figure 1A). Neurons flattened but still engulfed the NPs without being constrained by them (Figure 1A, inset). To observe the neuronal guidance on PDLO/NPs more precisely, high-magnification images were taken (Figure 1B). This displayed a detailed view of the guidance and growth of neuronal cell body and its neuritic projections, whereas (Figure 1C) demonstrated a lineup of a bundle of neuronal axons explicitly attached to the PDLO/NPs but not to the exterior planar substrate, albeit the substrate was also coated with PDLO. In the same fashion, this precise guidance of neuronal ensembles was also retained on a ring-shaped circle of PDLO/ NPs compared to the planar substrate coated with PDLO (Figure 1D). It should be noted that NPs consist of a thin polymer scaffold that improves their flexibility causing neurons and their protrusions to bend the pillars along the patterned line (Figure $1 \mathrm{~B}-\mathrm{D}$ ). In turn, this feature promotes the interaction between the cell membrane and PDLO/NPs because of the adaptation of NPs to forces exerted by cell attachment leading to tight adhesion rather than cell penetration. $^{40}$ Interestingly, our results demonstrate the ordering and alignment of neuronal ensembles provided by the combinatorial patterning effect of nanotopographical cues using 3D NPs and biochemical cues exerted by the PDLO. The observed cell guidance response to the competitive physical/ chemical interaction might promote tight proximity with the cell membranes that might favor particular localized extracellular stimuli, which in turn modulate a range of cellular signaling responses. ${ }^{41}$

3.2. Neuronal Morphological Characterization. We next examined the impact of different surface functionalizations (adhesion factors) in inducing patterned neuronal ensembles (Figure 2 and Supporting Information Figure S1). To do so, besides exploiting the PDLO molecule $(70 \mu \mathrm{g} / \mathrm{mL})$, before cell seeding, we functionalized the $3 \mathrm{D} \mathrm{NP}$ substrates with different adhesion-promoting molecules, including $0.01 \%$ PLO (SigmaAldrich), $50 \mu \mathrm{g} / \mathrm{mL}$ PLL(Sigma-Aldrich), and $100 \mu \mathrm{g} / \mathrm{mL}$ $\mathrm{PDL}$ (Sigma-Aldrich), that were also compared with a planar $\mathrm{Au}$ substrate coated with PLO, PLL, PDL, and PDLO. Hippocampal neuronal cultures were fixed in $4 \%$ paraformaldehyde and stained for MAP-2, a specific soma-dendritic neuronal marker as previously reported ${ }^{37}$ (see Methods). Analysis by fluorescence confocal microscopy showed a well-defined ordered array of neurons grown on PDLO/NPs (Figure 2A), indicated by the expression level of MAP-2 protein. This result was also confirmed by the intensity profile of a grid pattern of labeled MAP-2 neuronal protein, where the grid edge lines displayed the highest fluorescent intensity, whereas no fluorescence intensity was detected outside the grid pattern (Figure 2A). When other polycationic molecules were used to functionalize 3D NP substrate, we obtained clusters, and fasciculated neuronal processes on PLO/NPs, which were also confirmed by the fluorescence intensity profile (Figure 2B). Furthermore, neurons were grown on patterned grids of PLL/ NPs, and PDL/NP substrates yielded a nonspecific growth spanned on the patterned and the exterior planar regions without ordered array, which was also confirmed by the fluorescence intensity profile (Figure 2C,D). Furthermore, compelling evidence identified the significance of nanostructures to support and promote the growth of adherent cells as inferred by upregulating the formation of focal adhesion (FA) points on the surface between the nanostructures. ${ }^{40,42}$ Thus, we examined the effect of the nanotopographical cues (3D NPs) on cell guidance compared to planar $\mathrm{Au}$ substrates functionalized with PLO, PLL, PDL, and PDLO. This yielded similar results to those observed using $3 \mathrm{D} \mathrm{NP}$ substrates functionalized with promoting molecules, that is, PLO, PLL, and PDL, which displayed the paucity of cell guidance (random grown network) 

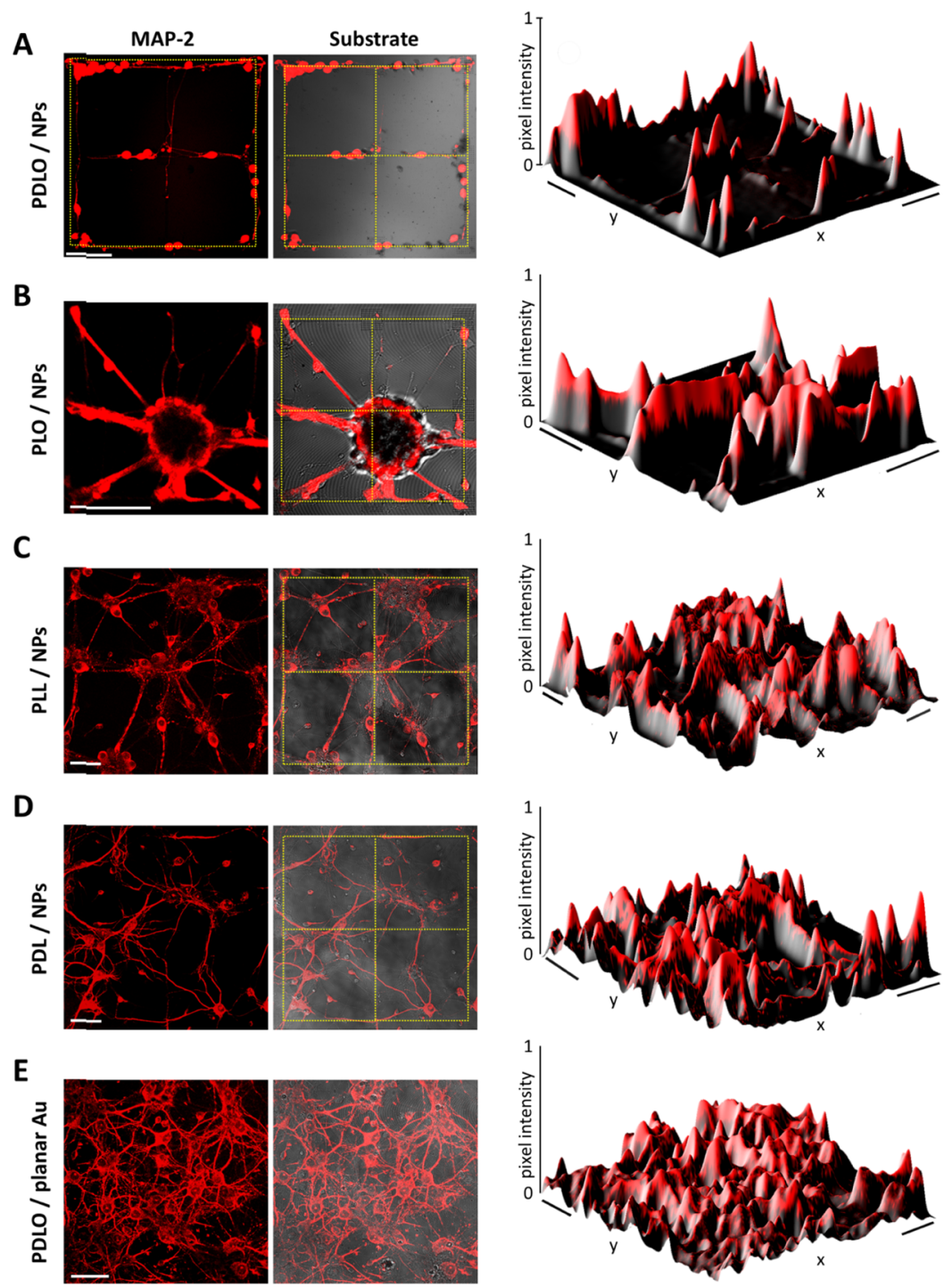

Figure 2. Fluorescence images displaying the reliance of neuronal morphological arrangement on combinatorial biochemical and physical cues. (A) Hippocampal neurons stained with MAP-2 and overlaid on 3D NP grid substrate showing a precise guidance on PDLO/NP grid pattern. The Intensity profile of neuronal MAP-2 fluorescence signal detected in neurons grown on PDLO/NP grid pattern. The yellow dotted grids denote the region of 3D NP array. (B) As in (A) but for neurons grown on PLO/NP substrate that evince the paucity of cell guidance and fasciculate neuronal processes. The intensity profile of MAP-2 fluorescence signal observed in neurons grown on PLO/NP grid pattern. (C) As in (A,B) but for neurons grown on PLL/NP substrate that evident a random nonpatterned neuronal growth. The intensity profile of neurons grown on PLL/NP grid pattern. (D) As in (C) but for random morphological arrangement and intensity profile of neurons grown on PDL/NP grid pattern. (E) Neurons grown on a planar Au substrate coated with PDLO display a random morphological arrangement. Scale bars represent $50 \mu \mathrm{m}$ for all images on the left. Scale bars represent $(40 \mu \mathrm{m} \times 40 \mu \mathrm{m}, 30 \mu \mathrm{m} \times 30 \mu \mathrm{m}, 70 \mu \mathrm{m} \times 70 \mu \mathrm{m}$, and $40 \mu \mathrm{m} \times 40 \mu \mathrm{m})$ for the intensity profiles from A to E, respectively.

on the pattern grids (Figure 2E and Supporting Information Figure S1).

To quantify our observations in achieving cell guidance on PDLO/NPs rather than any other physical and/or chemical cues, we analyzed the spatial orientation of neuronal cells by counting the cell bodies guided on patterned grids and nonguided on planar surfaces functionalized with PDLO, PLO, PLL, and PDL (Figure 3). To do so, we restricted the counted cells to bidirectional regions, that is, green and red ROI of $20 \mu \mathrm{m} \times 20 \mu \mathrm{m}$, which approximately emerged from the corner of each pattern grid (Figure 3A). Whenever cells were visualized within green ROIs, they were counted positive cells for the patterned group. Cells outside a given ROI were otherwise considered negative cells and assigned to the nonpatterned group. We found that when neurons were grown on PDLO/NPs, in average, $90 \%$ of those neurons were guided by the geometrical structure of the 3D NPs functionalized with PDLO, whereas only $10 \%$ were grown out of the pattern grid (Figure 3B). In contrast, when other biochemical molecules were used to functionalize the 3D NPs, a low number of neurons were found growing on the pattern grids conferred by $27.4,24.4$, and $20.3 \%$ for PLO, PLL, and PDL, respectively (Figure $3 \mathrm{~B}$ ). In addition, we disentangled the effects of 3D nanostructures from the effects of surface functionalization on cell guidance by quantifying neuronal cell bodies on a planar Au substrate coated with PDLO. This showed a lack of cell guidance analogous to results obtained from neurons grown on PLO, PLL, and PDL coated on 3D NPs (Figure 3B). Thus, our results confirm the selective orientation and preferential cell guidance to grow neuronal 


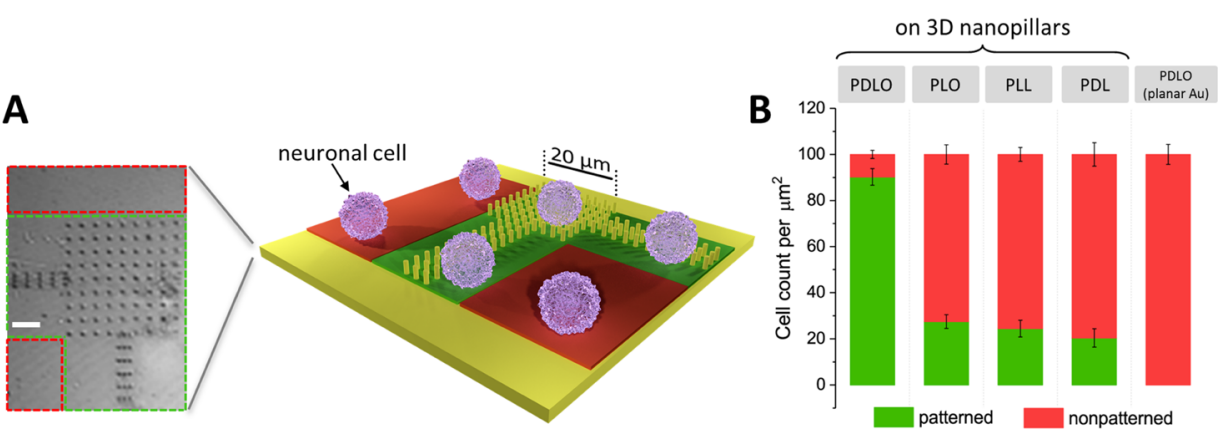

Figure 3. Quantification of neuronal cell guidance on 3D NP grid patterns and different surface functionalizations. (A) Schematic of the cell quantification method. (Left) A phase-contrast image of 3D NP grid pattern indicating a green ROI for counted patterned cells and red ROIs for nonpatterned cells. (Right) Graphical schematic emulates the counting method on 3D NPs. Scale bar represents $10 \mu \mathrm{m}$. (B) Normalized numbers of counted cells on guided-patterned and nonpatterned regions as functions of nanotopographical cues (3D NPs vs planar Au) and biochemical cues (PDLO, PLO, PLL, and PDL). Quantifications showing significantly $(90 \pm 3.5 \%)$ guided neurons grown on PDLO/NPs compared to (27.44 \pm $2.96,24.43 \pm 3.62$, and $20.37 \pm 3.99 \%$ ) of neurons grown on 3D NPs functionalized with PLO, PLL, and PDL, respectively. However, no guidance was yielded for neurons grown on planar Au functionalized with PDLO. The significant difference in measurements is assessed with one-way analysis of variance (ANOVA) followed by Tukey's post hoc testing. ( $n=4 ; p<0.01$; data expressed as mean \pm standard error of the mean).

A
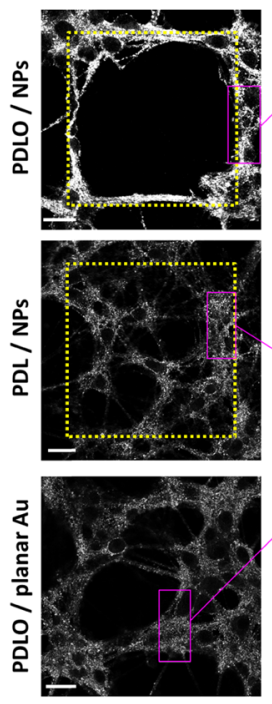

fluorescence
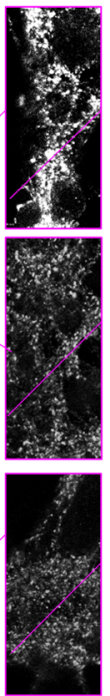

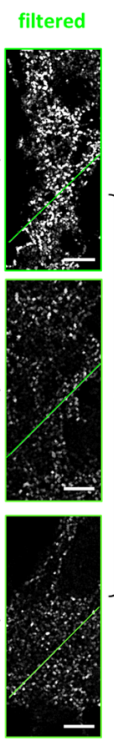

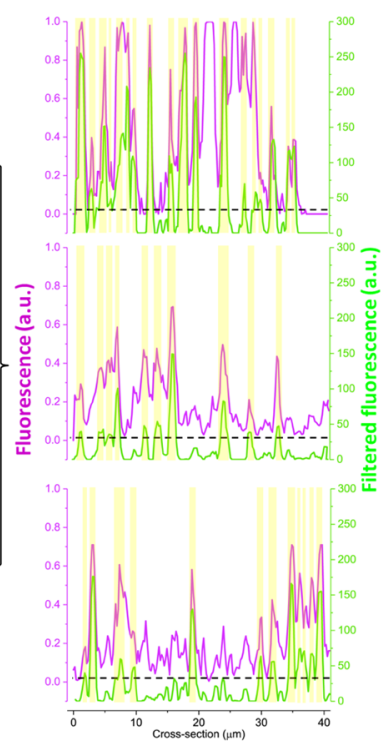

B

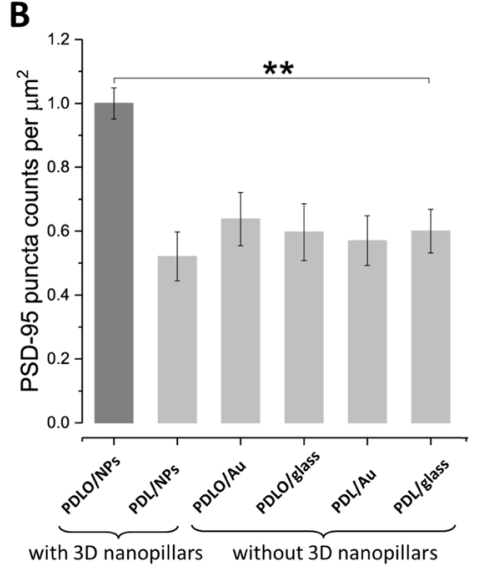

Figure 4. Quantification of combinatorial effects of nanotopographical and biochemical cues on synaptic maturation and stability. (A) (Left) Confocal micrographs displaying neuronal PSD-95 expression on 3D NPs functionalized with PDLO and PDL and on a planar Au surface functionalized with PDLO. (Right) Quantification of PSD-95 protein expression is performed by processing the images with the granulometric filter method. The magenta and green cross sections indicate the fluorescence and the corresponding filtered intensities, respectively. The start and the end positions of the cross sections are read from the left $(0 \mu \mathrm{m})$ to the right $(40 \mu \mathrm{m})$. The yellow dotted grids denote the regions of 3D NPs. The position of the PSD-95 puncta was defined above an arbitrary offset on the filtered fluorescence scale, that is, 30, indicated by the black dotted lines. All scale bars represent $30 \mu \mathrm{m}$ in images on the left and $10 \mu \mathrm{m}$ in images on the right. (B) Bar graph showing the quantified combinatorial effects of nanotopographical cues (3D NPs, planar $\mathrm{Au}$, and planar glass surfaces) and biochemical cues (PDLO and PDL) conferred by the normalized numbers of PSD-95 ensembles (density). PSD-95 puncta is significantly higher in neurons grown on PDLO/NPs $(1 \pm 0.048)$ compared to (0.52 \pm 0.076, $0.63 \pm 0.083,0.59 \pm 0.088,0.57 \pm 0.077$, and $0.59 \pm 0.068)$ for neurons grown on PDL/NPs, PDLO/planar Au, PDLO/planar glass, PDL/ planar $\mathrm{Au}$, and PDL/planar glass, respectively. $(n=4, * * p<0.01$, ANOVA).

processes along the isotropic arrangement of specific combinatorial PDLO/NP pattern grids.

3.3. Synaptic Characterization. We investigated the formation, maturation, and the stability of neuronal synaptic network in long-term cultures of self-ordered neurons guided on PDLO/NPs compared to other chemical/physical cues. To do so, we quantified the expression level of PSD-95 protein in neurons grown on multiple chemical/physical substrates (Figure 4A and Supporting Information Figure S2). Notably, PSD-95 is the major postsynaptic scaffold protein that is exclusively localized to glutamatergic synapses. ${ }^{43}$ PSD-95 drives synaptic maturation ${ }^{44}$ and promotes synapse stability ${ }^{45}$ by maintaining the physiological ratio of excitatory-to-inhibitory synapses in the network. ${ }^{46}$ PSD-95 expression was also shown to increase during in vitro neuronal development, which is, however, correlated with the increase of neuronal electrical activity in rodents ${ }^{47}$ and human-derived neurons. ${ }^{37}$ In addition, many etiologies of autism spectrum disorder and schizophrenia imply a disruption in synapses, particularly, altered PSD-95 expression in neuronal circuits. ${ }^{48}$ Although a recent study reported the effects of nanotopographical cues in the formation of functional connectivity and neuronal circuit activity, ${ }^{49}$ the 
A
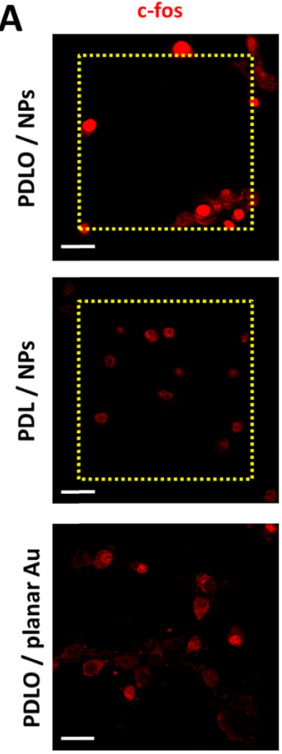

MAP-2/c-fos
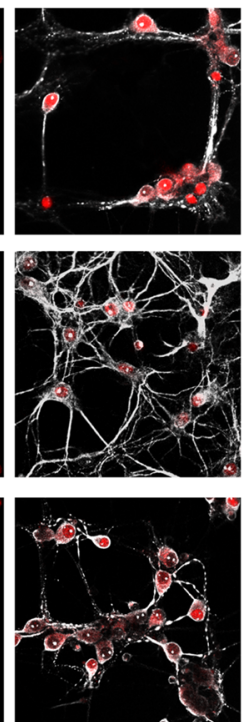

B

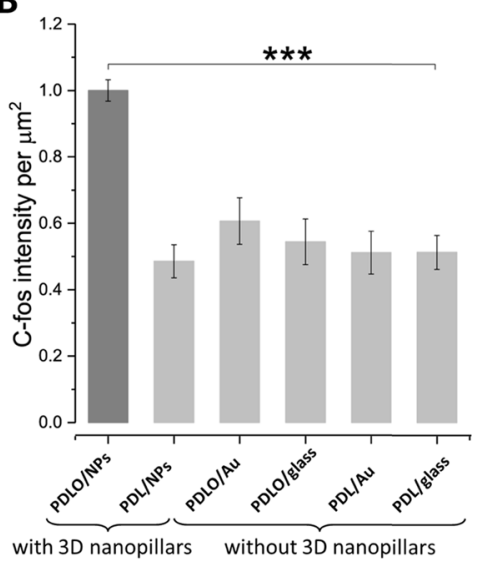

Figure 5. Quantification of combinatorial effects of nanotopographical and biochemical cues on cellular activity. (A) Confocal micrographs showing the c-fos and MAP-2 immunofluorescence of neurons grown on 3D NPs functionalized with PDLO and PDL and neurons grown on a planar Au surface functionalized with PDLO. The yellow dotted grids denote the regions of 3D NPs. Scale bars represent $30 \mu \mathrm{m}$. (B) Bar graph showing the quantified combinatorial effects of nanotopographical cues (3D NPs, planar Au, and planar glass surfaces) and biochemical cues (PDLO and PDL) conferred by the normalized fluorescence intensity of c-fos expression (functional cellular activity). C-fos activity is significantly higher in neurons grown on PDLO/NPs $(1 \pm 0.032)$ compared to $(0.48 \pm 0.049,0.6 \pm 0.069,0.54 \pm 0.068,0.51 \pm 0.064$, and $0.51 \pm 0.051)$ for neurons grown on $\mathrm{PDL} / \mathrm{NPs}, \mathrm{PDLO} / \mathrm{planar} \mathrm{Au}, \mathrm{PDLO} /$ planar glass, PDL/planar Au, and PDL/planar glass, respectively. $(n=4, * * * p<0.001, \mathrm{ANOVA})$.

effect of combined biochemical/physical cues on synapse stability remains undetermined.

Here, we used a confocal upright microscope with $25 \times / 0.95$ NA objective to acquire images from neurons grown on multiple chemical/physical substrates, that is, PDLO/NPs, $\mathrm{PDL} / \mathrm{NPs}, \mathrm{PDLO} /$ planar Au, PDLO/planar glass, PDL/planar $\mathrm{Au}$, and $\mathrm{PDL} /$ planar glass (Figure 4 and Supporting Information Figure S2). Then, granulometric filtering method was performed to quantify the PSD-95 assembles in various functionalization conditions as previously reported ${ }^{37}$ (see Methods). The magenta and green cross sections display the fluorescence and the corresponding filtered intensities, respectively (Figure $4 \mathrm{~A}$ ). We set an arbitrary offset, that is, a dashed line at 30 to pinpoint the PSD-95 puncta above that level, whereas the yellow bars represent the location of the puncta above the offset. Furthermore, we quantified the density of PSD-95 construct by using an automatic particle counting method integrated into the ImageJ platform. ${ }^{38}$ We analyzed the synaptic density by counting PSD-95 immunoreactive puncta per $\mu \mathrm{m}^{2}$, visualized in multiple fields of the images and multiple cultures. The results of these analyses showed a significantly higher intensity and density of PSD-95 constructs in the guided neurons on $\mathrm{PDLO} / \mathrm{NPs}$ compared to all other substrates (Figure 4B and Supporting Information Figure S2). This result suggests a significant role of our patterning method using PDLO/NP combination to regulate the synaptic activity through PSD-95 dynamics that might be critical in the activity-dependent remodeling of neuronal connections leading to neuronal maturation and the stabilization of synapses.

3.4. Cellular Activity Characterization. To investigate if neurons guided on a pattern grid of PDLO/NP substrates were also associated with an increased level of network activity, we further evaluated neuronal activity on different substrates. We assayed the cellular intensity of endogenous c-fos, a neuronal marker of the early activity-dependent gene. c-fos plays a vital role in synaptic functions induced by activity, which in turn impacts neuronal development and plasticity. ${ }^{50}$ Confocal images were acquired with $25 \times / 0.95 \mathrm{NA}$ objective of the upright microscope from neurons grown on multiple chemical/ physical substrates, that is, PDLO/NPs, PDL/NPs, PDLO/ planar $\mathrm{Au}, \mathrm{PDLO} /$ planar glass, $\mathrm{PDL} /$ planar $\mathrm{Au}$, and $\mathrm{PDL} /$ planar glass (Figure 5A and Supporting Information Figure S3). We quantified the intensity of c-fos immunofluorescence by using an automatic object counting method integrated into ImageJ platform (see Methods). We found a significantly higher expression of c-fos activity in neurons grown and guided on a pattern grid of PDLO/NPs compared to neurons grown with no guidance on substrates with NPs, planar Au, and glass in the presence of different adhesion-promoting molecules (Figure 5B and Supporting Information Figure S3). In summary, our results confirm at the cellular resolution the functional neuronal activity of a neuronal network guided by specific nanotopographical cues (NPs), when particularly functionalized with PDLO, that is, PDLO/NPs. The enhanced cellular activity of neurons grown on PDLO/NPs may confirm the distance-dependent fashion between neurons that has been found to influence the neuronal firing activity in vivo. ${ }^{51}$ Neurons guided on PDLO/NPs are closely located and thus have the higher possibility of developing synaptic connections with each other, compared to those located far from each other. This enhanced activity might be a result of the continual postsynaptic remodeling, which in turn promotes the growth and stabilization of synapses. ${ }^{47}$

\section{DISCUSSION}

This study demonstrates the use of the combinatorial nanotopographical (3D NP arrays) and biochemical cues (PDLO) to generate well-defined patterned arrays of neuronal ensembles and to support their functional features. In turn, the guided neurons on PDLO/NPs exhibit enhanced synaptic 
formation and maturation leading to synapse stability and network cellular activity. These results identify a new cell patterning technique that could reveal the synaptic dynamics of functional multicellular neuronal cultures at a high spatial resolution in a large-scale network and might provide insight into the control of neuronal polarization and growth. Although substrates containing hierarchical structures of nanoscale cues allow for controlled guidance through the simultaneous modulation of the cytoskeleton and intracellular FA protein assembly, ${ }^{52}$ the precise mechanism by which PDLO/NPs interact with neuronal ensembles and foster guidance remains to be determined. Notably, several lines of evidence indicate the substantial effect of topography features at both nano and microscales on cell adhesion and guidance cues throughout the redistribution of FA complexes and integrins. ${ }^{8}$ For instance, integrin clustering is initiated upon interactions between a cell and the ECM that in turn leads to the manifestation of FAs through a positive feedback mechanism. ${ }^{28}$ In addition, FAs and integrin form focal sites serving to function as molecular checkpoints for transmitting external signals from the ECM to the cell nucleus. Accordingly, a specific effect of PDLO on promoting cell adhesion could be associated with the structure of the ornithine molecule formed already in the high molecular weight of the polycationic substance (PDLO), and also the additional carbonyl groups, which impart higher negative charges, leading to an increase in the binding sites for tight cell attachment. This evidence suggests that the PDLO/NP combination forming cellular focal points is very likely triggering a downstream signaling through the actin-binding proteins that leads to the recruitment of FA contact in the dynamic cytoskeleton complexes, which in turn foster the adhesion of neurons to the underlying physical cues (3D NPs). The presence of these adhesion constructs could promote a tight seal between the cell membrane and multiple NPs, suggesting that the modulation of cellular adhesion can alter the NP-cell interface. ${ }^{53}$ In turn, this would provide detailed spatial resolution across neuronal ensembles to monitor synaptic activity that is critical for understanding cell signaling and drug development in neurophysiological and pathological conditions. Furthermore, another interesting theory that may be tempting to speculate on the significance of PDLO is stereochemistry of the molecule that influences cellular behavior on a substrate and hence affects the in vitro cell chirality. ${ }^{54}$ Chirality, that is, L/ D configurations, is a distinct biochemical signature, where most amino acids exist as L-enantiomers, and many biological and physiological processes are greatly influenced by the conformation of proteins and other biomacromolecules. ${ }^{55}$ Therefore, a distinct cell adhesion may result from a specific chiral configuration rather than others. Although $\mathrm{L}$ form, such as in PLL and PLO, was shown to enhance the adsorption of ECM protein to the substrate, ${ }^{56} \mathrm{D}$ form such as in PDL was reported to be more resistant than $\mathrm{L}$ form to proteases produced by cells in cultures. ${ }^{57}$ Interestingly, PDLO exhibits a racemic mixture of L/D forms, which might set a balance of top-notch chiral properties (enzymatic degradation vs protection) required for neurons to adhere, grow, spread, and assemble on nanotopographical cues better than other molecules that possess a distinct L or D configuration. Consequently, the chirality of the PDLO molecule may cause a marked stimulation to cell surface receptors to enhance protein adsorption on the cell membrane leading to the exposure of specific epitopes for cell adhesion. On the other hand, chiral structures could be distributed distinctly on the cell membrane, where their overall behavior enables the generation of significant biophysical signals that can be translated into local symmetric gene expression. In turn, this suggests that PDLO conformation and the spatial distribution of active sites may play a vital role in the neurite-promoting function using PDLO/NPs. Therefore, our combinatorial platform using PDLO/NPs could be used as a new paradigm to elucidate the role of intracellular machinery of cell chirality in neuronal development and diseases. However, to confirm the precise role of cellular adhesion elements in the ordered array of guided neurons, the implications on the functional development of neural ensembles requires further studies.

\section{CONCLUSIONS}

In this work, we reported the use of surface chemistry combined with nanostructured substrates as a new tool to further explore, in vitro, the proper set of physical/chemical neuronal guidance cues required to promote synaptic maturation, and we demonstrated that this could lead to enhanced synapse stability and cellular activity in neural ensembles. We introduced a simple yet powerful approach in which a specific adhesion-promoting molecule (PDLO) is linked to the surface of 3D NPs-(PDLO/NPs) to foster an ordered array of guided hippocampal neuronal ensembles. By using SEM and confocal imaging, we demonstrated, for the first time, that only synergistic topographical and biochemical cues (PDLO/NPs) could yield 90\% guided neurons in well-defined pattern grids. Importantly, we found that our method enhanced synaptic formation and maturation and thus promoted synapse stability in cells guided on pattern grids, as indicated by the increased level of PSD-95 expression in neurons grown on PDLO/NPs. We also confirmed that neurons grown on a pattern grid of $\mathrm{PDLO} / \mathrm{NP}$ substrates generated higher functional cellular activity indicated by c-fos expression compared to neurons grown on planar or surfaces functionalized with other biochemical molecules, which in turn associated with the synaptic maturation and stability.

Significantly, the outcome of this study will contribute to our knowledge of neurons grown on chemically modified 3D nanostructured substrates by fostering synergistic interplay between the fields of nanoscience, biochemistry, and neuroscience, which could revolutionize future neuroprosthetic and therapeutic applications. Furthermore, it provides a quantitative tool to gauge progress on improving nanostructures for biomanipulation of neuronal networks at the level of synapse. Prospectively, our method can be exploited to engineer a chipbased electrophysiological approach to simultaneously monitor the electrical and synaptic dynamics of guided neuronal ensembles on functionalized 3D NPs that are integrated into multielectrode arrays. By extension, our method would enable multifunctional recording of extracellular, intracellular, ${ }^{31}$ and biochemical signals in a highly localized and targeted fashion by the exploitation of plasmonic enhancement of NPs. ${ }^{39}$

\section{ASSOCIATED CONTENT}

\section{Supporting Information}

The Supporting Information is available free of charge on the ACS Publications website at DOI: 10.1021/acsami.8b00387.

Fluorescence images displaying neuronal morphological arrangement on planar $\mathrm{Au}$ surface precoated with biochemical adhesion molecules; quantification of combinatorial effects of nanotopographical and biochemical cues on synaptic maturation and stability; and 
quantification of combinatorial effects of nanotopographical and biochemical cues on cellular activity (PDF)

\section{AUTHOR INFORMATION}

\section{Corresponding Author}

*E-mail: hayder.amin@iit.it.

\section{ORCID $\odot$}

Hayder Amin: 0000-0002-6515-2991

Michele Dipalo: 0000-0002-1823-8231

Francesco De Angelis: 0000-0001-6053-2488

\section{Author Contributions}

The manuscript was prepared through contributions of all authors. All authors have given approval to the final version of the manuscript.

\section{Notes}

The authors declare no competing financial interest.

\section{ACKNOWLEDGMENTS}

This work was partly supported by the European Commission for Research within the Seventh Framework Program for the NAMASEN (FP7-264872) Marie-Curie ITN and partly by the European Research Council under the European Union's Seventh Framework Programme (FP/2007-2013)/ERC grant agreement no. (616213), CoG: Neuro-Plasmonics. We would like to thank Dr. Steven Moore (University of Cambridge) for his valuable comments and contribution in editing the manuscript to improve clarity and readability.

\section{REFERENCES}

(1) Kandel, E. R.; Schwartz, J. H.; Jessell, T. M. Principles of Neural Science; McGraw-Hill, Health Professions Division, 2000.

(2) Barral, J.; Reyes, A. D. Synaptic Scaling Rule Preserves Excitatory-inhibitory Balance and Salient Neuronal Network Dynamics. Nat. Neurosci. 2016, 19, 1690-1696.

(3) Yuste, R. From the Neuron Doctrine to Neural Networks. Nat. Rev. Neurosci. 2015, 16, 487-497.

(4) Amin, H.; Nieus, T.; Lonardoni, D.; Maccione, A.; Berdondini, L. High-Resolution Bioelectrical Imaging of $\mathrm{A} \beta$-Induced Network Dysfunction on CMOS-MEAs for Neurotoxicity and Rescue Studies. Sci. Rep. 2017, 7, 2460.

(5) Palop, J. J.; Mucke, L. Network Abnormalities and Interneuron Dysfunction in Alzheimer Disease. Nat. Rev. Neurosci. 2016, 17, 777792.

(6) Stam, C. J. Modern Network Science of Neurological Disorders. Nat. Rev. Neurosci. 2014, 15, 683-695.

(7) Garzón-Muvdi, T.; Quiñones-Hinojosa, A. Neural Stem Cell Niches and Homing: Recruitment and Integration into Functional Tissues. ILAR J. 2009, 51, 3-23.

(8) Dowell-Mesfin, N. M.; Abdul-Karim, M.-A.; Turner, A. M. P.; Schanz, S.; Craighead, H. G.; Roysam, B.; Turner, J. N.; Shain, W. Topographically Modified Surfaces Affect Orientation and Growth of Hippocampal Neurons. J. Neural. Eng. 2004, 1, 78-90.

(9) Dalby, M. J. Topographically Induced Direct Cell Mechanotransduction. Med. Eng. Phys. 2005, 27, 730-742.

(10) Park, T. H.; Shuler, M. L. Integration of Cell Culture and Microfabrication Technology. Biotechnol. Prog. 2003, 19, 243-253.

(11) Li, W.; Tang, Q. Y.; Jadhav, A. D.; Narang, A.; Qian, W. X.; Shi, P.; Pang, S. W. Large-Scale Topographical Screen for Investigation of Physical Neural-Guidance Cues. Sci. Rep. 2015, 5, 8644.

(12) Offenhäusser, A.; Böcker-Meffert, S.; Decker, T.; Helpenstein, R.; Gasteier, P.; Groll, J.; Möller, M.; Reska, A.; Schäfer, S.; Schulte, P.; Vogt-Eisele, A. Microcontact Printing of Proteins for Neuronal Cell Guidance. Soft Matter 2007, 3, 290-298.
(13) Vidu, R.; Rahman, M.; Mahmoudi, M.; Enachescu, M.; Poteca, T. D.; Opris, I. Nanostructures: A Platform for Brain Repair and Augmentation. Front. Syst. Neurosci. 2014, 8, 91.

(14) Conway, A.; Vazin, T.; Spelke, D. P.; Rode, N. A.; Healy, K. E.; Kane, R. S.; Schaffer, D. V. Multivalent Ligands Control Stem Cell Behaviour in Vitro and in Vivo. Nat. Nanotechnol. 2013, 8, 831-838.

(15) Marconi, E.; Nieus, T.; Maccione, A.; Valente, P.; Simi, A.; Messa, M.; Dante, S.; Baldelli, P.; Berdondini, L.; Benfenati, F. Emergent Functional Properties of Neuronal Networks with Controlled Topology. PLoS One 2012, 7, No. e34648.

(16) Hobbs, R. G.; Petkov, N.; Holmes, J. D. Semiconductor Nanowire Fabrication by Bottom-up and Top-down Paradigms. Chem. Mater. 2012, 24, 1975-1991.

(17) Kim, M.-H.; Park, M.; Kang, K.; Choi, I. S. Neurons on Nanometric Topographies: Insights into Neuronal Behaviors in Vitro. Biomater. Sci. 2014, 2, 148-155.

(18) Xie, X.; Xu, A. M.; Leal-Ortiz, S.; Cao, Y.; Garner, C. C.; Melosh, N. A. Nanostraw-Electroporation System for Highly Efficient Intracellular Delivery and Transfection. ACS Nano 2013, 7, 43514358.

(19) Na, Y.-R.; Kim, S. Y.; Gaublomme, J. T.; Shalek, A. K.; Jorgolli, M.; Park, H.; Yang, E. G. Probing Enzymatic Activity inside Living Cells Using a Nanowire-Cell "sandwich" assay. Nano Lett. 2013, 13, 153-158.

(20) Silberberg, Y. R.; Kawamura, R.; Ryu, S.; Fukazawa, K.; Ishihara, K.; Nakamura, C. Detection of Microtubules in Vivo Using AntibodyImmobilized Nanoneedles. J. Biosci. Bioeng. 2014, 117, 107-112.

(21) McKnight, T. E.; Melechko, A. V.; Griffin, G. D.; Guillorn, M. A.; Merkulov, V. I.; Serna, F.; Hensley, D. K.; Doktycz, M. J.; Lowndes, D. H.; Simpson, M. L. Intracellular Integration of Synthetic Nanostructures with Viable Cells for Controlled Biochemical Manipulation. Nanotechnology 2003, 14, 551-556.

(22) VanDersarl, J. J.; Xu, A. M.; Melosh, N. A. Nanostraws for Direct Fluidic Intracellular Access. Nano Lett. 2012, 12, 3881-3886.

(23) Shalek, A. K.; Gaublomme, J. T.; Wang, L.; Yosef, N.; Chevrier, N.; Andersen, M. S.; Robinson, J. T.; Pochet, N.; Neuberg, D.; Gertner, R. S.; Amit, I.; Brown, J. R.; Hacohen, N.; Regev, A.; Wu, C. J.; Park, H. Nanowire-Mediated Delivery Enables Functional Interrogation of Primary Immune Cells: Application to the Analysis of Chronic Lymphocytic Leukemia. Nano Lett. 2012, 12, 6498-6504.

(24) Xie, C.; Hanson, L.; Xie, W.; Lin, Z.; Cui, B.; Cui, Y. Noninvasive Neuron Pinning with Nanopillar Arrays. Nano Lett. 2010, $10,4020-4024$

(25) Santoro, F.; Schnitker, J.; Panaitov, G.; Offenhäusser, A. On Chip Guidance and Recording of Cardiomyocytes with 3D Mushroom-Shaped Electrodes. Nano Lett. 2013, 13, 5379-5384.

(26) Wheeler, B. C.; Brewer, G. J. Designing Neural Networks in Culture. Proc. IEEE 2010, 98, 398-406.

(27) Bouton, C. E.; Shaikhouni, A.; Annetta, N. V.; Bockbrader, M. A.; Friedenberg, D. A.; Nielson, D. M.; Sharma, G.; Sederberg, P. B.; Glenn, B. C.; Mysiw, W. J.; Morgan, A. G.; Deogaonkar, M.; Rezai, A. R. Restoring Cortical Control of Functional Movement in a Human with Quadriplegia. Nature 2016, 533, 247-250.

(28) Nguyen, A. T.; Sathe, S. R.; Yim, E. K. F. From Nano to Micro: Topographical Scale and Its Impact on Cell Adhesion, Morphology and Contact Guidance. J. Phys. Condens. Matter 2016, 28, 183001.

(29) Kang, K.; Park, Y.-S.; Park, M.; Jang, M. J.; Kim, S.-M.; Lee, J.; Choi, J. Y.; Jung, D. H.; Chang, Y.-T.; Yoon, M.-H.; Lee, J. S.; Nam, Y.; Choi, I. S. Axon-First Neuritogenesis on Vertical Nanowires. Nano Lett. 2016, 16, 675-680.

(30) Hai, A.; Shappir, J.; Spira, M. E. In-Cell Recordings by Extracellular Microelectrodes. Nat. Methods 2010, 7, 200-202.

(31) Dipalo, M.; Amin, H.; Lovato, L.; Moia, F.; Caprettini, V.; Messina, G. C.; Tantussi, F.; Berdondini, L.; De Angelis, F. Intracellular and Extracellular Recording of Spontaneous Action Potentials in Mammalian Neurons and Cardiac Cells with 3D Plasmonic Nanoelectrodes. Nano Lett. 2017, 17, 3932-3939. 
(32) Abbott, J.; Ye, T.; Qin, L.; Jorgolli, M.; Gertner, R. S.; Ham, D.; Park, H. CMOS Nanoelectrode Array for All-Electrical Intracellular Electrophysiological Imaging. Nat. Nanotechnol. 2017, 12, 460-466.

(33) Liu, R.; Chen, R.; Elthakeb, A. T.; Lee, S. H.; Hinckley, S.; Khraiche, M. L.; Scott, J.; Pre, D.; Hwang, Y.; Tanaka, A.; Ro, Y. G.; Matsushita, A. K.; Dai, X.; Soci, C.; Biesmans, S.; James, A.; Nogan, J.; Jungjohann, K. L.; Pete, D. V.; Webb, D. B.; Zou, Y.; Bang, A. G.; Dayeh, S. A. High Density Individually Addressable Nanowire Arrays Record Intracellular Activity from Primary Rodent and Human Stem Cell Derived Neurons. Nano Lett. 2017, 17, 2757-2764.

(34) Duan, X.; Gao, R.; Xie, P.; Cohen-Karni, T.; Qing, Q.; Choe, H. S.; Tian, B.; Jiang, X.; Lieber, C. M. Intracellular Recordings of Action Potentials by an Extracellular Nanoscale Field-Effect Transistor. Nat. Nanotechnol. 2012, 7, 174-179.

(35) Kundu, A.; Micholt, L.; Friedrich, S.; Rand, D. R.; Bartic, C.; Braeken, D.; Levchenko, A. Superimposed Topographic and Chemical Cues Synergistically Guide Neurite Outgrowth. Lab Chip 2013, 13, 3070.

(36) De Angelis, F.; Malerba, M.; Patrini, M.; Miele, E.; Das, G.; Toma, A.; Zaccaria, R. P.; Di Fabrizio, E. 3D Hollow Nanostructures as Building Blocks for Multifunctional Plasmonics. Nano Lett. 2013, 13, 3553-3558.

(37) Amin, H.; Maccione, A.; Marinaro, F.; Zordan, S.; Nieus, T.; Berdondini, L. Electrical Responses and Spontaneous Activity of Human iPS-Derived Neuronal Networks Characterized for 3-Month Culture with 4096-Electrode Arrays. Front. Neurosci. 2016, 10, 121.

(38) Schneider, C. A.; Rasband, W. S.; Eliceiri, K. W. NIH Image to Image J: 25 Years of Image Analysis. Nat. Methods 2012, 9, 671-675.

(39) Dipalo, M.; Messina, G. C.; Amin, H.; La Rocca, R.; Shalabaeva, V.; Simi, A.; Maccione, A.; Zilio, P.; Berdondini, L.; De Angelis, F. 3D Plasmonic Nanoantennas Integrated with MEA Biosensors. Nanoscale 2015, 7, 3703-3711.

(40) Xie, X.; Xu, A. M.; Angle, M. R.; Tayebi, N.; Verma, P.; Melosh, N. A. Mechanical Model of Vertical Nanowire Cell Penetration. Nano Lett. 2013, 13, 6002-6008.

(41) Ingber, D. E. Cellular Mechanotransduction: Putting All the Pieces Together Again. FASEB J. 2006, 20, 811-827.

(42) Bonde, S.; Berthing, T.; Madsen, M. H.; Andersen, T. K.; BuchMånson, N.; Guo, L.; Li, X.; Badique, F.; Anselme, K.; Nygård, J.; Martinez, K. L. Tuning InAs Nanowire Density for HEK293 Cell Viability, Adhesion, and Morphology: Perspectives for NanowireBased Biosensors. ACS Appl. Mater. Interfaces 2013, 5, 10510-10519.

(43) Hunt, C. A.; Schenker, L. J.; Kennedy, M. B. PSD-95 Is Associated with the Postsynaptic Density and Not with the Presynaptic Membrane at Forebrain Synapses. J. Neurosci. 1996, 16, $1380-1388$.

(44) El-Husseini, A. E.; Schnell, E.; Chetkovich, D. M.; Nicoll, R. A.; Bredt, D. S. PSD-95 Involvement in Maturation of Excitatory Synapses. Science 2000, 290, 1364-1368.

(45) Taft, C. E.; Turrigiano, G. G. PSD-95 Promotes the Stabilization of Young Synaptic Contacts. Philos. Trans. R. Soc., B 2013, 369, 20130134

(46) Prange, O.; Wong, T. P.; Gerrow, K.; Wang, Y. T.; El-Husseini, A. A Balance between Excitatory and Inhibitory Synapses Is Controlled by PSD-95 and Neuroligin. Proc. Natl. Acad. Sci. U.S.A. 2004, 101, 13915-13920.

(47) Statman, A.; Kaufman, M.; Minerbi, A.; Ziv, N. E.; Brenner, N. Synaptic Size Dynamics as an Effectively Stochastic Process. PLoS Comput. Biol. 2014, 10, No. e1003846.

(48) de Bartolomeis, A.; Latte, G.; Tomasetti, C.; Iasevoli, F. Glutamatergic Postsynaptic Density Protein Dysfunctions in Synaptic Plasticity and Dendritic Spines Morphology: Relevance to Schizophrenia and Other Behavioral Disorders Pathophysiology, and Implications for Novel Therapeutic Approaches. Mol. Neurobiol. 2014, 49, 484-511.

(49) Gautam, V.; Naureen, S.; Shahid, N.; Gao, Q.; Wang, Y.; Nisbet, D.; Jagadish, C.; Daria, V. R. Engineering Highly Interconnected Neuronal Networks on Nanowire Scaffolds. Nano Lett. 2017, 17, $3369-3375$.
(50) Hevroni, D.; Rattner, A.; Bundman, M.; Lederfein, D.; Gabarah, A.; Mangelus, M.; Silverman, M. A.; Kedar, H.; Naor, C.; Kornuc, M.; Hanoch, T.; Seger, R.; Theill, L. E.; Nedivi, E.; Richter-Levin, G.; Citri, Y. Hippocampal Plasticity Involves Extensive Gene Induction and Multiple Cellular Mechanisms**. J. Mol. Neurosci. 1998, 10, 75-98.

(51) Miri, A.; Daie, K.; Arrenberg, A. B.; Baier, H.; Aksay, E.; Tank, D. W. Spatial Gradients and Multidimensional Dynamics in a Neural Integrator Circuit. Nat. Neurosci. 2011, 14, 1150-1159.

(52) Keselowsky, B. G.; Collard, D. M.; García, A. J. Surface Chemistry Modulates Focal Adhesion Composition and Signaling through Changes in Integrin Binding. Biomaterials 2004, 25, 59475954.

(53) Xu, A. M.; Aalipour, A.; Leal-Ortiz, S.; Mekhdjian, A. H.; Xie, X.; Dunn, A. R.; Garner, C. C.; Melosh, N. A. Quantification of Nanowire Penetration into Living Cells. Nat. Commun. 2014, 5, 3613.

(54) Wan, L. Q.; Chin, A. S.; Worley, K. E.; Ray, P. Cell Chirality: Emergence of Asymmetry from Cell Culture. Philos. Trans. R. Soc., B 2016, 371, 20150413.

(55) Inaki, M.; Liu, J.; Matsuno, K. Cell Chirality: Its Origin and Roles in Left-Right Asymmetric Development. Philos. Trans. R. Soc., B 2016, 371, 20150403.

(56) Banker, G.; Goslin, K. Culturing Nerve Cells; MIT Press, 1998.

(57) Tugyi, R.; Uray, K.; Iván, D.; Fellinger, E.; Perkins, A.; Hudecz, F. Partial D-Amino Acid Substitution: Improved Enzymatic Stability and Preserved Ab Recognition of a MUC2 Epitope Peptide. Proc. Natl. Acad. Sci. U.S.A. 2005, 102, 413-418. 